Journal of Mathematical Physics, Analysis, Geometry

2015, vol. 11, No. 2, pp. 174-186

\title{
On Model Representations of Non-Selfadjoint Operators with Infinitely Dimensional Imaginary Component
}

\author{
R. Hatamleh \\ Dept. of Mathematics, Jadara University \\ Irbid-Jordan, Jordan \\ E-mail: raedhat@yahoo.com \\ V.A. Zolotarev \\ B. Verkin Institute for Low Temperature Physics and Engineering \\ National Academy of Sciences of Ukraine \\ ${ }_{47} 7$ Lenin Ave., Kharkiv 61103, Ukraine \\ E-mail: vazolotarev@gmail.com
}

Received September 9, 2013, revised December 26, 2014

For an entirely non-selfadjoint operator with spectrum at zero, the imaginary component of which has an absolutely continuous spectrum (not necessarily dissipative and having lacunas in the spectrum), triangular and functional models are constructed.

Key words: triangular model, simple spectrum, colligation, functional model.

Mathematics Subject Classification 2010: 47A45.

The subject of the paper is the generalization of the well-known result obtained by M. S. Livšic for the case of infinitely dimensional imaginary component $[1,3]$.

Livšic's Theorem 1. Let $A$ be an entirely non-selfadjoint $[2,3]$ operator in a Hilbert space $H$ such that

a) the spectrum of the operator $A$ is concentrated at zero, $\sigma(A)=\{0\}$;

b) $A$ is dissipative, and its imaginary component is one-dimensional, $\operatorname{rank} A_{I}=1$.

Then the operator $A$ is unitarily equivalent to the integration operator $\widetilde{A}$,

$$
(\widetilde{A} f)(x)=i \int_{x}^{l} f(t) d t
$$

acting in the space $L_{[0, l]}^{2}$, where $l \neq 0$ is an eigenvalue of $2 A_{I}=\frac{A-A^{*}}{i}$.

(c) R. Hatamleh and V.A. Zolotarev, 2015 
In what follows, an analogue of this theorem is obtained for the case where the imaginary component $A_{I}$ of the operator $A$ is an operator with a simple absolutely continuous spectrum, also a functional model of this class of operators is constructed.

The generalization of the result of Livšic is based on the study of the operator of integration in the spaces of square summable functions on compacts in $\mathbb{R}_{+}^{2}$. It is stated that the imaginary component of the operator of integration depends on the choice of configuration of corresponding compacts. It can be non-dissipative, can have lacunas in the spectrum, can have simple spectrum, etc.

The first result generalizing Livšic's Theorem 1 obtained in [7] is Theorem 5, where the compact $\Omega_{L}$ has a triangular form and is bounded by a smooth decreasing curve $L$ and by the lines $x=a, y=b$. Since Theorems $2-5$ of Sections I, II are proved in [7], they are listed here without proofs. The development of the scheme of the constructions of Sections I, II is given in Sections III-V. Thus, for a non-dissipative operator with an imaginary component having a simple absolutely continuous spectrum, an analogue of theorem of Livšic (Theorem 7) is obtained, where the compact $\Omega_{L}$ is represented by conjugation of two triangular domains. In Section V, Theorem 8 (an analogue of Livšic's theorem) is proved, where the imaginary component of the dissipative operator is supposed to have a lacuna in the absolutely continuous spectrum. The compact $\Omega_{L}$ has a triangular form, and the smooth curve $L$ possesses constant values on some inner interval. The proved Theorems $5,7,8$ show wide possibilities for triangular models due to the choices of a compact $\Omega_{L}$.

It turns out that the functional model for the given classes of operators can be constructed in Hilbert spaces representing symbiosis of $L^{2}$ spaces and WienerPaley spaces of entire functions.

I. Consider a continuous curve $L$ in $\mathbb{R}_{+}^{2}$,

$$
L=\left\{(x, y): y=\alpha(x) \in C_{[0, a]}^{1} ; \alpha(0)=b, \alpha(a)=0\right\},
$$

generated by a smooth monotonically decreasing function $\alpha:[0, a] \rightarrow \mathbb{C}(0<a$, $b<\infty)$. Denote by $\Omega_{L}$ a compact in $\mathbb{R}_{+}^{2}$ bounded by the curve $L(2)$ and the lines $x=a, y=b$. Define the Hilbert space $L_{\Omega_{L}}^{2}$ formed by the complex-valued square summable functions $f: \Omega_{L} \rightarrow \mathbb{C}$,

$$
L_{\Omega_{L}}^{2}=\left\{f: \iint_{\Omega_{L}}|f(x, y)|^{2} d x d y<\infty\right\} .
$$


Denote by $\widetilde{A}$ the linear bounded operator acting in $L_{\Omega_{L}}^{2}(3)$ :

$$
(\widetilde{A} f)(x, y) \stackrel{\text { def }}{=} i \int_{x}^{a} f(t, y) d t \text {. }
$$

It is easy to see that

$$
2\left(\widetilde{A}_{I} f\right)(x, y)=\chi_{\Omega_{L}} \int_{\alpha^{-1}(y)}^{a} f(t, y) d t
$$

where $\alpha^{-1}(y)$ is a smooth monotonically decreasing function on $[0, b]$ inverse to $\alpha(x)\left(\alpha^{-}(\alpha(x))=x, \forall x \in[0, a]\right)$, and $\chi_{\Omega_{L}}$ is a characteristic function of the set $\Omega_{L}$. Equality (5) implies that

$$
G \stackrel{\text { def }}{=} \overline{\widetilde{A}_{I} L_{\Omega_{L}}^{2}}=\left\{f \chi_{\Omega_{L}} \in L_{\Omega_{L}}^{2}\right\}
$$

where $f:[0, b] \rightarrow \mathbb{C}$ is a complex-valued function of the variable $y$.

Specify the smooth non-negative monotonically increasing function

$$
\lambda(y) \stackrel{\text { def }}{=} a-\alpha^{-1}(y) \quad(y \in[0, b]) .
$$

Since

$$
\iint_{\Omega_{L}}\left|f(y) \chi_{\Omega_{L}}\right|^{2} d x d y=\int_{0}^{b}|f(y)|^{2} \lambda(y) d y,
$$

the subspace $G(6)$ is isomorphic to the weighted space

$$
L_{(0, b)}^{2}(\lambda(y) d y) \stackrel{\text { def }}{=}\left\{f: \int_{0}^{b}|f(y)|^{2} \lambda(y) d y<\infty\right\} .
$$

The mapping

$$
U: G \rightarrow L_{(0, b)}^{2}(\lambda(y) d y) ; \quad(U f)(y) \chi_{\Omega_{L}} \stackrel{\text { def }}{=} f(y)
$$

defines the bi-unique correspondence between $G(6)$ and $L_{(0, b)}^{2}(\lambda(y) d y)$. It is obvious that $U$ is unitary. Formula (5) yields that the operator $\tilde{\sigma}=\left.U 2 A_{I}\right|_{G} U^{*}$ in the space $L_{(0, b)}^{2}(\lambda(y) d y)$ acts as a multiplication by the function $\lambda(y)(7)$,

$$
(\widetilde{\sigma} f)(y) \stackrel{\text { def }}{=} \lambda(y) f(y)
$$


where $f \in L_{(0, b)}^{2}(\lambda(y) d y)$. It is easy to see that the orthoprojector $P_{G}$ on the subspace $G(6)$ is given by

$$
\left(P_{G} f\right)(x, y) \stackrel{\text { def }}{=} \frac{\chi_{\Omega_{L}}}{\lambda(y)} \int_{\alpha^{-1}(y)}^{a} f(t, y) d t .
$$

Map now the operator $\widetilde{\varphi}$ from $L_{\Omega_{L}}^{2}(3)$ onto $L_{(0, b)}^{2}(\lambda(y) d y)(8)$ by the formula

$$
\widetilde{\varphi}=U P_{G},
$$

where $P_{G}$ is given in (11). As a result, we obtain that the family

$$
\widetilde{\Delta}=\left(\widetilde{A}, L_{\Omega_{L}}^{2}, \widetilde{\varphi}, L_{(0, b)}^{2}(\lambda(y) d y), \widetilde{\sigma}\right)
$$

is a colligation $[2,3]$, where the operators $\widetilde{A}, \widetilde{\varphi}$ and $\widetilde{\sigma}$ are given by (4), (12), and (10), respectively.

Theorem 2. [7] The characteristic function $S_{\widetilde{\Delta}}(z)[1-3]$ of the colligation $\widetilde{\Delta}$ (13) is a scalar operator in $L_{(0, l)}^{2}(\lambda(y) d y)$,

$$
\left(S_{\widetilde{\Delta}}(z) f\right)(y)=e^{\frac{i \lambda(y)}{z}} f(y),
$$

where $\lambda(y)$ is given by (7), and $f(y) \in L_{(0, l)}^{2}(\lambda(y) d y)$.

The proof of the theorem is given in [7].

$\mathrm{O}$ b s e r v a t i o $\mathrm{n} 1$. The operator-function $S_{\widetilde{\Delta}}(z)(14)$ commutes with the operator $\widetilde{\sigma}(10)$ for all $z \in \mathbb{C}(z \neq 0)$.

II. Consider a bounded selfadjoint operator $B$ with a simple spectrum given in a Hilbert space $H$. The spectrum of the operator $B$ belongs to the interval $[0, a]$. Then, according to [6], the operator $B$ is unitarily equivalent to the operator of multiplication by the independent variable

$$
(\widehat{B} f)(\lambda)=\lambda f(\lambda), \quad f(\lambda) \in L_{(0, a)}^{2}(d \sigma(\lambda),
$$

where $\sigma(\lambda)=\left\langle E_{\lambda} u, u\right\rangle$ is a non-decreasing function on $[0, a], E_{\lambda}$ is the resolution of identity of $B$, and $u \in H$ is the generating vector of the operator $B$. Suppose that the measure $d \sigma(\lambda)$ is absolutely continuous by the Lebesgue measure

$$
d \sigma(\lambda)=m(\lambda) d \lambda, \quad m(\lambda)=\sigma^{\prime}(\lambda) \geq 0 .
$$

Definition 1. [7] An absolutely continuous measure $d \sigma(\lambda), \lambda \in[0, a]$ from (16) is said to have the $A C_{0}$-property if

$$
\int_{0}^{\lambda} \frac{d \sigma(t)}{t}<\infty
$$


for all $\lambda \in[0, a]$.

Condition (17) requires that the improper integral converge at zero. Define the positive monotonically increasing function $y(\lambda)$,

$$
y(\lambda) \stackrel{\text { def }}{=} \int_{0}^{\lambda} \frac{d \sigma(t)}{t} .
$$

$\mathrm{O} \mathrm{b} \mathrm{s}$ e $\mathrm{r} \mathrm{v}$ a t i o $\mathrm{n} 2$. 'A priori' we can assume that the function $y(\lambda)$ maps $[0, a]$ onto $[0, b]$, where $b$ is the preassigned finite positive number. If $y(\lambda)$ : $[0, a] \rightarrow[0, d](d>0)$, then, specifying the measure $d \sigma_{1}(\lambda)=\frac{b}{d} d \sigma(\lambda), b>0$, and substituting $f(\lambda) \rightarrow \sqrt{\frac{d}{b}} f(\lambda)$ in $L_{(0, a)}^{2}(d \sigma(\lambda))$, we obtain the Hilbert space $L_{(0, a)}^{2}\left(d \sigma_{1}(\lambda)\right)$ isomorphic to $L_{(0, a)}^{2}(d \sigma(\lambda))$. Besides, the function $y_{1}(\lambda)$ constructed by $d \sigma_{1}(\lambda)$ (20) already possesses the values belonging to $[0, b]$. This procedure signifies the renormalization of the generating vector $u \rightarrow \sqrt{\frac{b}{d}} u$ since $\sigma(\lambda)=\left\langle E_{\lambda} u, u\right\rangle$.

Denote by $\lambda(y)$ the function inverse to $y(\lambda)(18)(y(\lambda(y))=y, \forall y)$. Since $d \sigma(\lambda)=\lambda d y(\lambda)$, then the change of the variable $\lambda \rightarrow \lambda(y)$ transforms the space $L_{(0, a)}^{2}(d \sigma(\lambda))$ in $L_{(0, b)}^{2}(\lambda(y) d y)$, in which the operator $\widehat{B}(15)$ acts as a multiplication by the function $\lambda(y)$,

$$
(\widetilde{B} f)(y)=\lambda(y) f(y),
$$

where $f(y) \in L_{(0, b)}^{2}(\lambda(t) d y)$.

Theorem 3. [7] Let $B$ be a bounded selfadjoint operator with a simple spectrum in $H$, and the spectrum of $B$ belongs to the segment $[0, a]$. If the spectral measure $\sigma(\lambda)$ of the operator $B$ is absolutely continuous (16) and has the $A C_{0}$ property (17), then the operator $B$ is unitarily equivalent to the operator of multiplication $\widetilde{B}(19)$ by the smooth monotonically increasing function $\lambda(y)$ (inverse to $y(\lambda)(18))$ in $L_{(0, b)}^{2}(\lambda(y) d y)$, where the finite positive number $b$ can be chosen arbitrarily.

The following statement gives the description of the commutant of the operator $\widehat{B}(17)$.

Theorem 4. [7] An arbitrary linear operator $\widehat{A}$ in $L_{(0, a)}^{2}(d \sigma(\lambda))$ commuting with $\widehat{B}(15)$ is the multiplication operator

$$
(\widehat{A} f)(\lambda)=a(\lambda) f(\lambda)
$$


where $f(\lambda) \in L_{(0, a)}^{2}(d \sigma(\lambda))$, and $a(\lambda)$ is a complex-valued function from $L_{(0, a)}^{2}(d \sigma(\lambda))$, and $\|A\|=\|a(\lambda)\|_{L_{(0, a)}^{2}(d \sigma(\lambda))}$.

The proof of the theorem is given in [7].

The following statement generalizing the result of Livšic's Theorem 1 is true.

Theorem 5. [7] Let a linear bounded dissipative completely non-selfadjoint operator $A$ with spectrum at zero, $\sigma(A)=\{0\}$, be given in a Hilbert space $H$ and

(1) let the operator $2 A_{I}$ restricted to $H_{1}=\overline{A_{I} H}$ have a simple spectrum filling the segment $[0, a], 0<a<\infty$, and let its spectral function $\sigma(\lambda)$ be absolutely continuous (16) and have the $A C_{0}$-property (17);

(2) for all $z \in \mathbb{C}, z \neq 0$, let $\left[P_{H_{1}}(A-z I)^{-1} P_{H_{1}}, A_{I}\right]=0$ take place, where $P_{H_{1}}$ is an orthoprojector on $H_{1}$.

Then the operator $A$ is unitarily equivalent to the integration operator $\widetilde{A}$,

$$
(\widetilde{A} f)(x, y)=i \int_{x}^{a} f(t, y) d t
$$

in the space $L_{\Omega_{L}}^{2}(3)$, where the curve $L(2)$ is given by the function $\alpha^{-1}(y)=$ $a-\lambda(y)$ and $\lambda(y)$ is inverse to the function $y(\lambda)(18)$.

This theorem is proved in [7].

In Sections III-V we will use the constructions of Sections I, II.

III. Similarly to (2), consider the curve $L$ in $\mathbb{R}_{+}^{2}$,

$$
L=\left\{(x, y): y=\alpha(x) \in C_{[0, c]}^{1} ; \alpha(0)=b, \alpha(c)=0\right\},
$$

where $\alpha:[0, c] \rightarrow \mathbb{C}$ is a smooth monotonically decreasing function on $[0, c], 0<b$, $c<\infty$. Let $a$ be a point lying between 0 and $c, 0<a<c$. Denote by $\Omega_{L}$ the compact in $\mathbb{R}_{+}^{2}$ consisting of two linked components, $\Omega_{L}=\Omega_{L}^{1} \cup \Omega_{L}^{2}$. Thus, $\Omega_{L}^{1}$ represents the domain bounded by the curve $L$ and lines $y=b, x=a ; \Omega_{L}^{2}$ is bounded, correspondingly, by the curve $L$ and the lines $y=0, x=a$. Define the Hilbert space $L_{\Omega_{L}}^{2}(3)$ for the given $\Omega_{L}$. Specify in $L_{\Omega_{L}}^{2}$ the linear bounded operator

$$
(\widetilde{A} f)(x, y) \stackrel{\text { def }}{=} i \int_{x}^{a} f(t, y) d t,
$$

where $f \in L_{\Omega_{L}}^{2}$ (3). Just as in the case of the operator $\widetilde{A}(4)$, it is easy to show that for $\widetilde{A}(23)$

$$
2\left(\widetilde{A}_{I} f\right)(x, y)=\chi_{\Omega_{L}} \int_{\alpha^{-1}(y)}^{a} f(t, y) d t
$$


takes place, where $\chi_{\Omega_{L}}$ is the characteristic function of the set $\Omega_{L}$ and $\alpha^{-1}(y)$ is the inverse function to $\alpha(x)\left(\alpha^{-1}(\alpha(x))=x \forall x \in[0, c]\right)$. Similarly to (6), we have that

$$
G \stackrel{\text { def }}{=} \widetilde{\widetilde{A}_{I} L_{\Omega_{L}}^{2}}=\left\{f \chi_{\Omega_{L}} \in L_{\Omega_{L}}^{2}\right\},
$$

where $f:[0, b] \rightarrow \mathbb{C}$ is a function of the variable $y$. Specify the smooth monotonically increasing function

$$
\lambda(y) \stackrel{\text { def }}{=} a-\alpha^{-1}(y) .
$$

Obviously, this function maps the segment $[0, b]$ on $[a-c, a]$, where $\lambda(d)=0$, $d=y(a)$. Thus the function $\lambda(y)$ on the segment $[0, d]$ possesses the values from $[a-c, 0]$ and on $[d, b]$, correspondingly, from $[0, a]$.

Since

$$
\begin{aligned}
\iint_{\Omega_{L}}\left|f(y) \chi_{\Omega_{L}}\right|^{2} d x d y & =\int_{d}^{b}|f(y)|^{2} \lambda(y) d y+\int_{0}^{d}|f(y)|^{2}(-\lambda(y)) d y \\
& =\int_{0}^{b}|f(y)|^{2}|\lambda(y)| d y
\end{aligned}
$$

the subspace $G(25)$ is isomorphic to the weighted space

$$
L_{(0, b)}^{2}(|\lambda(y)| d y) \stackrel{\text { def }}{=}\left\{f: \int_{0}^{b}|f(y)|^{2}|\lambda(y)| d y<\infty\right\} .
$$

The unitary correspondence between $G(25)$ and $L_{(0, b)}^{2}(|\lambda(y)| d y)(27)$ is realized by the map

$$
U: G \rightarrow L_{(0, b)}^{2}(|\lambda(y)| d y) ; \quad(U f)(y) \chi_{\Omega_{L}} \stackrel{\text { def }}{=} f(y) .
$$

The operator $\widetilde{\sigma}=U 2 \widetilde{A}_{I} U^{*}$ in the space $L_{(0, b)}^{2}(|\lambda(y)| d y)$ acts by means of multiplication by the function $\lambda(y)(26)$,

$$
(\widetilde{\sigma} f)(y) \stackrel{\text { def }}{=} \lambda(y) f(y)
$$

where $f \in L_{(0, b)}^{2}(|\lambda(y)| d y)$. It is easy to show that the orthoprojector $P_{G}$ on the subspace $G(25)$ is equal to

$$
\left(P_{G} f\right)(x, y) \stackrel{\text { def }}{=} \frac{\chi_{\Omega_{L}}}{\lambda(y)} \int_{\alpha^{-1}(y)}^{a} f(t, y) d t .
$$


Specify the operator $\widetilde{\varphi}: L_{\Omega_{L}} \rightarrow L_{(0, b)}^{2}(|\lambda(y)| d y)$ by the formula

$$
\widetilde{\varphi}=U P_{G}
$$

where $P_{G}$ is given by (30). Thus, the family

$$
\widetilde{\Delta}=\left(\widetilde{A}, L_{\Omega_{L}}^{2}, \widetilde{\varphi}, L_{(0, b)}^{2}(|\lambda(y)| d y), \widetilde{\sigma}\right)
$$

is a colligation in which the operators $\widetilde{A}, \widetilde{\varphi}$, and $\widetilde{\sigma}$ are given by (23), (31), and (29), respectively.

Theorem 6. The characteristic function $S_{\widetilde{\Delta}}(z)$ of the colligation $\widetilde{\Delta}(32)$ acts in $L_{(0, b)}^{2}(|\lambda(y)| d y)$ as follows:

$$
\left(S_{\widetilde{\Delta}}(z) f\right)(y)=e^{\frac{i \lambda(y)}{z}} f(y)
$$

where $\lambda(y)$ is given by $(26)$, and $f \in L_{(0, b)}^{2}(|\lambda(y)| d y)$.

The proof of Theorem 6 is similar to that of Theorem 2 [7].

As in the previous case, it is obvious that

$$
S_{\widetilde{\Delta}}(z) \widetilde{\sigma}=\widetilde{\sigma} S_{\widetilde{\Delta}}(z)
$$

for all $z \in \mathbb{C}(z \neq 0)$.

IV. Analogously to the considerations of Section II, every bounded selfadjoint operator $B$ with simple spectrum such that $\sigma(B) \subset[\alpha, \beta](-\infty<\alpha<0<\beta<$ $\infty)$ is unitarily equivalent to the operator $\widehat{B}$

$$
(\widehat{B} f(\lambda))=\lambda f(\lambda)
$$

where $f(\lambda) \in L_{(\alpha, \beta)}^{2}(d \sigma(\lambda))$. The spectral measure $d \sigma(\lambda)$ is supposed to be absolutely continuous (16).

Definition 2. An absolutely continuous measure $d \sigma(\lambda)(\lambda \in[\alpha, \beta])(16)$ is said to have the $A C_{0}$-property if

$$
\int_{\alpha}^{\lambda} \frac{d \sigma(t)}{|t|}<\infty
$$

for all $\lambda \in[\alpha, \beta]$. 
Similarly to (18), define the positive monotonically increasing function

$$
y(\lambda)=\int_{\alpha}^{\lambda} \frac{d \sigma(t)}{|t|}
$$

mapping the segment $[\alpha, \beta]$ on $[0, b]$ where $0<b<\infty$. Denote by $\lambda(y)$ the function inverse to $y(\lambda)(36)$. From (36) we have that

$$
\lambda d y(\lambda)=d \sigma(\lambda) \quad(\lambda \in[0, \beta]) ; \quad-\lambda d y(\lambda)=d \sigma(\lambda) \quad(\lambda \in[\alpha, 0]) .
$$

Therefore, after changing the variable $\lambda \rightarrow \lambda(y)$, we obtain that the space $L_{(\alpha, \beta)}^{2}(d \sigma(\lambda))$ is isomorphic to the space $L_{(0, b)}^{2}(|\lambda(y)| d y)$, in which the operator $\widehat{B}$ acts as an operator of multiplication by the function $\lambda(y)$,

$$
(\widetilde{B} f)(y)=\lambda(y) f(y)
$$

where $f(y) \in L_{(0, b)}^{2}(|\lambda(y)| d y)$.

It is obvious that in this case the following analogue of Theorem 3 is true.

The considerations stated above allow us to formulate the statement of Theorem 7 (similar to Theorem 6) without supposing that the initial operator $A$ is dissipative.

Theorem 7. Let $A$ be a linear completely non-selfadjoint operator acting in a Hilbert space $H$ with spectrum at zero, $\sigma(A)=\{0\}$. Then

(1) the operator $2 A_{I}$ restricted on $H_{1}=\overline{A_{I} H}$ has a simple spectrum filling the segment $[a-c, a](0<a<c<\infty)$, and its spectral function $\sigma(\lambda)$ is absolutely continuous (19) and has the $A C_{0}$-property (35);

(2) for all $z \in \mathbb{C}(z \neq 0),\left[P_{H_{1}}(A-z I)^{-1} P_{H_{1}}, A_{I}\right]=0$ takes place, where $P_{H_{1}}$ is the orthoprojector on $H_{1}$.

Hence the operator $A$ is unitarily equivalent to the operator of integration $\widetilde{A}$,

$$
(\widetilde{A} f)(x, y)=i \int_{x}^{a} f(t, y) d t
$$

in the space $L_{\Omega_{L}}^{2}(3)$, and the curve $L(22)$ is given by the function $\alpha^{-1}(y)=$ $a-\lambda(y)$, where $\lambda(y)$ is the function inverse to $y(\lambda)(36), \lambda(y)=y^{-1}(\lambda)$.

P r o o f. As follows from the above considerations, there exists the unitary operator $U: H_{1} \rightarrow L_{(0, b)}^{2}(|\lambda(y)| d y)$ such that $U 2 A_{I}=\widetilde{B} U$, where $\widetilde{B}$ is given by (37). Construct the colligation

$$
\Delta=\left(A, H, U P_{H_{1}}, L_{(0, b)}^{2}(|\lambda(y)| d y), \widetilde{B}\right)
$$


where $P_{H_{1}}$ is the orthoprojector on $H_{1}$. Condition (2) of the theorem yields that the characteristic function $S_{\Delta}(z)$ of the colligation $\Delta$ commutes with the operator $\widetilde{B}$. Applying Theorem 4, we obtain that $S_{\Delta}(z)$ is the operator of multiplication by the function $\exp \left\{i z^{-1} c(y)\right\}$ in the space $L_{(0, b)}^{2}(|\lambda(y)| d y)$ in view of the known form of the characteristic function if one takes into account that $\sigma(A)=\{0\}$. It is easy to see that $c(y)=\lambda(y)$ since $\lim _{z \rightarrow \infty} i z\left(1-S_{\Delta}(z)\right)=\widetilde{B}$.

Knowing $\lambda(y)$, by using formula $(26)$, we can construct the smooth decreasing function $\alpha^{-1}(y)$ mapping $[0, b]$ onto $[0, c]$, besides, if $y(0)=d, 0<d<b$, then $x(d)=a$. Using $x(y)$, we specify the curve $L(22)$ and construct the domain $\Omega_{L}$. Next define the colligation $\widetilde{\Delta}(32)$, where $L_{\Omega_{L}}^{2}$ is given by (3) and the operators $\widetilde{A}, \widetilde{\varphi}$, and $\widetilde{\sigma}$ are given by (23), (31), and (29), respectively. To conclude the proof, it is left to note that the characteristic functions of the colligations $\Delta$ and $\widetilde{\Delta}$ coincide in view of $(33)$.

V. Consider a piecewise decreasing curve

$$
\begin{gathered}
L=\left\{(x, y): y=\alpha(x) \in C_{\left[0, a_{3}\right]}^{1} ; \alpha(0)=b_{2}, \alpha\left(a_{3}\right)=0 ;\right. \\
\left.\alpha(x)=b_{1}\left(\forall x \in\left[a_{1}, a_{2}\right]\right)\right\},
\end{gathered}
$$

where $0<a_{1}<a_{2}<a_{3}(<\infty), 0<b_{1}<b_{2}(<\infty)$, and the function $\alpha(x)$ decreases monotonically on the intervals $\left[0, a_{1}\right]$ and $\left[a_{2}, a_{3}\right]$. In the same way as in Section I, consider the compact $\Omega_{L}$ in $\mathbb{R}_{+}^{2}$ bounded by the curve $L(42)$ and the lines $x=a_{3}, y=b_{2}$. Denote by $L_{\Omega_{L}}^{2}$ (3) the Hilbert space of square summable functions on the compact $\Omega_{L}$. In $L_{\Omega_{L}}^{2}$, set the operator $\widetilde{A}$ by formula (4). Then, similarly to $(5)\left(a=a_{3}\right)$, we have

$$
2\left(\widetilde{A}_{I} f\right)(x, y)=\chi_{\Omega_{L}} \int_{\alpha^{-1}(y)}^{a_{3}} f(t, y) d t
$$

where $\alpha^{-1}:\left[0, b_{2}\right] \rightarrow \mathbb{R}$ is a monotonically decreasing function

$$
\alpha^{-1}(y)= \begin{cases}\alpha_{1}^{-1}(y), & y \in\left[b_{2}, b_{1}\right] \\ \alpha_{2}^{-1}(y), & y \in\left[0, b_{1}\right]\end{cases}
$$

where $\alpha_{1}^{-1}(\alpha(x))=x$ for all $x \in\left[0, a_{1}\right]$ and $\alpha_{2}^{-1}(\alpha(x))=x$ for all $x \in\left[a_{2}, a_{3}\right]$. One ought to consider ambiguity of the function $\alpha^{-1}(y)$ at the point $y=b_{1}$ as corresponding limits from the left for $\alpha_{2}^{-1}(y)$ ( from the right for $\alpha_{1}^{-1}(y)$ ) as $y \rightarrow b_{1}-0$ (correspondingly, as $y \rightarrow b_{1}+0$ ). Similarly to (7), we define a non-negative piecewise non-decreasing function

$$
\lambda(y)=a_{3}-\alpha^{-1}(y) \quad\left(y \in\left[0, b_{2}\right]\right) .
$$


Using $\lambda(y)(41)$, we define the space $L_{(0, b)}^{2}(\lambda(y) d y)(8)$ and the selfadjoint operator $\widetilde{\sigma}(10)$. In this case, the spectrum of the operator $\widetilde{\sigma}$ consists of two disjoint sets $\left[0, a_{3}-a_{2}\right] \cup\left[a_{3}-a_{1}, a_{3}\right]$ and has a lacuna $\left(a_{3}-a_{2}, a_{3}-a_{1}\right)$. As a result, we obtain the operator colligation

$$
\widetilde{\Delta}=\left(\widetilde{A}, L_{\Omega_{L}}^{2}, \widetilde{\varphi}, L_{(0, b)}^{2}(\lambda(y) d y), \widetilde{\sigma}\right),
$$

where $\widetilde{\varphi}$ and $\widetilde{\sigma}$ are given by (11), (12) and (10), respectively.

Theorem 2 is true. However, in representation (14) for the characteristic function $S_{\widetilde{\Delta}}(\lambda)$ of colligation (42), $\lambda(y)$ is given by (41) and $\alpha^{-1}(y)$, correspondingly, by $(40)$.

Repeating the considerations of Sections I, II, below we can get the analogue of Theorem 5 .

Theorem 8. Let $A$ be a linear bounded dissipative completely non-selfadjoint operator acting in a Hilbert space $H$ with spectrum at zero, $\sigma(A)=0$. Then

(1) the restriction of the operator $2 A_{I}$ on $H_{1}=\overline{A_{I} H}$ is an operator with a simple spectrum filling the set $\left[0, a_{3}-a_{2}\right] \cup\left[a_{3}-a_{1}, a_{3}\right], 0<a_{1}<a_{2}<a_{3}<\infty$, and its spectral function is absolutely continuous on this set and has the $A C_{0}$ property (20);

(2) for every $z \in \mathbb{C}, z \neq 0,\left[P_{H_{1}}(A-z I)^{-1} P_{H_{1}}, A_{I}\right]=0$ takes place, where $P_{H_{1}}$ is an orthoprojector on $H_{1}$.

Then the operator $A$ is unitarily equivalent to the operator $\widetilde{A}$

$$
(\widetilde{A} f)(x, y)=i \int_{x}^{a_{3}} f(t, y) d t
$$

in the space $L_{\Omega_{L}}^{2}(3)$, where the curve $L$ is given by (39) and $\alpha^{-1}(y)$ by (41), $\lambda(y)$ being the inverse function of $y(\lambda)(21)$.

VI. Let us turn to the construction of the functional model of the colligation $\widetilde{\Delta}(13)$. First, realize the Fourier transform by the variable $x$,

$$
F(z, y) \stackrel{\text { def }}{=} \mathcal{F}_{x} f(x, y)=\frac{1}{\sqrt{2 \pi}} \int_{\alpha^{-1}(y)}^{a} e^{i z x} f(x, y) d x
$$

The function $F(z, y)(44)$ is an entire function of exponential type by the variable $z$, the adjoint indicator diagram of which coincides with the segment of the imaginary axis $\left(i \alpha^{-1}(y), i a\right)$ [4]. As a result, we obtain the Hilbert space formed by the linear manifold of the functions $F(z, y)$ satisfying the conditions:

(a) $F(z, y)$ is an entire function of exponential type by $z$ having the adjoint diagram $\left(i \alpha^{-1}(y), i a\right)$ where $\alpha^{-1}(y)$ corresponds to the curve $L(2)$; 
(b) the inequality

$$
\int_{0}^{b} d y \int_{\mathbb{R}} d z|F(z, y)|^{2}<\infty
$$

takes place. Denote this Hilbert space by $W_{L}^{2}$. The Wiener-Paley and Plancherel Theorems $[4,5]$ imply that the transform $\mathcal{F}_{x}(47)$ defines the unitary isomorphism between the spaces $L_{\Omega_{L}}^{2}(3)$ and $W_{L}^{2}$.

Calculate the Fourier transform (44) of the operator $\widetilde{A}(4)$,

$$
\begin{gathered}
\mathcal{F}_{x}(\widetilde{A} f)(x, y)=\frac{1}{\sqrt{2 \pi}} \int_{\alpha^{-1}(y)}^{a} e^{i z x} i \int_{x}^{a} f(t, y) d t d x=\frac{i}{\sqrt{2 \pi}} \int_{\alpha^{-1}(y)}^{a} d t f(t, y) \int_{\alpha^{-1}(y)}^{t} e^{i z x} d x \\
=\frac{i}{\sqrt{2 \pi}} \int_{\alpha^{-1}(y)}^{a} d t f(t, y) \frac{e^{i z t}-e^{i z \alpha^{-1}(y)}}{i z}=\frac{1}{z}\left\{F(z, y)-e^{i z \alpha^{-1}(y)} F(0, y)\right\} .
\end{gathered}
$$

Thus, the operator $\widetilde{A}(4)$ in the space $W_{L}^{2}$ acts as follows:

$$
(\widehat{A} F)(z, y) \stackrel{\text { def }}{=} \frac{1}{z}\left\{F(z, y)-e^{i z \alpha^{-1}(y)} F(0, y)\right\},
$$

where $F \in W_{L}^{2}$. The Fourier transform (44) maps the space $G(6)$ onto the space

$$
\widetilde{G} \stackrel{\text { def }}{=}\left\{\frac{e^{i z a}-e^{i z \alpha^{-1}(y)}}{i z} f(y): f \in L_{(0, b)}^{2}(\lambda(y) d y)\right\} .
$$

Taking into account (11) and (44), we obtain that the orthoprojector $P_{\widetilde{G}}$ in the space $W_{L}^{2}$ on $\widetilde{G}$ is given by

$$
\left(P_{\widetilde{G}} F\right)(z, y) \stackrel{\text { def }}{=} \sqrt{2 \pi} \frac{e^{i z a}-e^{i z \alpha^{-1}(y)}}{i z \lambda(y)} F(0, y) .
$$

The operator $\widehat{\varphi}$, which is unitarily equivalent to $\widetilde{\varphi}(12)$, is equal to

$$
(\widehat{\varphi} F)(z, y) \stackrel{\text { def }}{=} \sqrt{2 \pi} \frac{F(0, y)}{\lambda(y)} .
$$

It is obvious that the adjoint operator $\widehat{\varphi}^{*}$ acting from $L_{(0, b)}^{2}(8)$ into $W_{L}^{2}$ is given by

$$
\left(\widehat{\varphi}^{*} f\right)(y)=\sqrt{2 \pi} \frac{e^{i z a}-e^{i z x(y)}}{i z} f(y) .
$$


Thus, we obtain the colligation

$$
\widehat{\Delta}=\left(\widehat{A}, W_{L}^{2}, \widehat{\varphi}, L_{(0, b)}^{2}(\lambda(y) d y), \widetilde{\sigma}\right),
$$

where the spaces $W_{L}^{2}$ and $L_{(0, b)}^{2}(\lambda(y) d y)$ are defined above and the operators $\widehat{A}$, $\widehat{\varphi}$, and $\widehat{\sigma}$ are given by (45), (48), and (10), respectively.

Theorem 9. Let $A$ be a linear completely non-selfadjoint dissipative operator acting in a Hilbert space $H$, the spectrum of which is concentrated at zero, $\sigma(A)=$ $\{0\}$. Then

(1) the operator $2 A_{I}$ restricted on $H_{1}=\overline{A_{I} H}$ has a simple spectrum filling the segment $[0, a], 0<a<\infty$, and its spectral function $\sigma(\lambda)$ is absolutely continuous and has the $A C_{0}$-property (20);

(2) for all $z \in \mathbb{C}, z \neq 0,\left[P_{H_{1}}(A-z I)^{-1} P_{H_{1}}, A_{I}\right]=0$ takes place, where $P_{H_{1}}$ is the orthoprojector on $H_{1}$.

Then the operator $A$ is unitarily equivalent to the operator $\widehat{A}$

$$
(\widehat{A} F)(z, y)=\frac{1}{z}\left\{F(z, y)-e^{i z \alpha^{-1}(y)} F(0, y)\right\}
$$

in the space $W_{L}^{2}$, where the curve $L(2)$ is given by the function $\alpha^{-1}(y), \lambda(y)$ is the function inverse to $y(\lambda)$ from (21).

Theorem 9 can be proved for the case of the domain $\Omega_{L}$ considered in Sections III, V.

\section{References}

[1] M.S. Livšic, On Spectral Decomposition of Linear Non-Selfadjoint Operators. Matem. sb. 34/76 (1954), No. 1, 145-198. (Russian)

[2] M.S. Livšic and A.A. Yantsevich, Theory of Operator Colligations in Hilbert Spaces. Kharkov University Publishing House, Kharkov, 1971. (Russian)

[3] V.A. Zolotarev, Analitic Methods of Spectral Representations of Non-Selfadjoint and Nonunitary Operators. Kharkov University Publishing House, Kharkov, 2003. (Russian)

[4] B.Ya. Levin, Lectures on Entire Functions. Amer. Math. Soc. Providence, RI, 150, 1996.

[5] N.I. Akhiezer, Lectures on Integral Transforms. Kharkov University Publishing House, Kharkov, 1984. (Russian)

[6] N.I. Akhiezer and I.M. Glazman, Theory of Linear Operators in Hilbert Space. Vol. 1, Vol. 2. Kharkov University Publishing House, Kharkov, 1978. (Russian)

[7] R. Hatamleh and V.A. Zolotarev, On Many-Dimensional Model Representations of One Class of Commuting Operators. - Ukr. Math. Jornal 66 (2014), No. 1, $108-127$. 